\title{
A NEW CALIBRATION METHOD FOR THE GEL PERMEATION CHROMATOGRAPHIC FRACTIONATION OF POLYMERS
}

\author{
SHyH-Gong SU(蘇世用) AND ChUng YUAN Mou(牟中原) \\ Department of Chemistry, National Taiwan University, \\ Taipei, Taiwan 107, R.O.C.
}

Key Word Index-Gel permeation chromatography; Fractionation of polymer.

\begin{abstract}
A model for the mechanism of the Gel permeation chromatographic separation of a polymer and a new calibration method have been developed, based on the exclusion of the polymer from the pores of the packing material. We propose that the calibration curve a plot of $\log (1-V K)$ versus $\log$ (molecular weight), where $K$ is the elution volume coefficient, is close to linear over a $K$ range of 0.15-0.85. We compare our procedure with that of Haller for various sets of data. Our procedure is generally better with lower standard deviations. When extrapolated, it gives a critical permeation size characteristic of the glass column packings.
\end{abstract}

Gel Permeation Chromatography has become a very useful instrument for polymer fractionation. The advent of new packing materials like controlled pore glass and covalent surface derivatization have allowed good separation of species of different molecular weights along with small band-spreading"). The rigidity and chemical inertness of these siliceous materials have made the results more reproducible and thus susceptible to a theoretical investigation of the mechanism. Therefore, a more accurate calibration method based on theoretical considerations would be very helpful toward the popularization of these methods. Determination of molecular weight by GPC usually requires standard samples for calibration, a better calibration method would certainly reduce the demand for expensive standards.

In GPC, the experimentally measured quantity is the elution volume, $V_{\text {e, for a }}$ polymer. Usually it is expressed in terms of the volumetric distribution ratio $K$ defined by

$$
V_{i}=V_{0}+V_{i} K
$$

where $V_{0}$ is the intestitial volume for the gel bed and $V_{i}$ is the internal volume for the gel bed. $V_{0}$ is obtained as the elution volume of an extremely large polymer excluded from the entire internal volume.

In the past, many calibration methods have been proposed for relating $K$ to the molecular weight $M$. Early attemps sought to plot molecular weight versus elution volume. Later, the logarithm of molecular weight was plotted against elution volume ${ }^{2,3)}$. Attempts to use molecular dimension parameters other than $M$, such as the diffusion constant, intrinsic viscosity, etc., have appeared from time to $t^{\prime} \mathrm{m}^{t, 3)}$. Most of them are limited in the range of application; usually $0.2<K<0.8$.

Recently, Haller") has used a plot of $\log (1-K)$ vs. $\log M$ with dextrans on controlled porous glass, and the plot is linear over the range $0.25<K<0.85$. This treatment was further refined by Basedow et $a l^{(5)}$ and Haller et $a l^{6}{ }^{\circ}$.

In a $\log M v s$. $K$ plot, the result is usually a " $S$ " shaped curve which makes the calibration more difficult. It would be interesting to further modify Haller's procedure such that linearity is obtained over the entive range of $K$. We propose in this paper a new procedure using $\log (1-\sqrt{K})$ as the plotting parameter. We 
show that our method gives better linearity. a large amount of independent data collected in the literature.

We begin, in the next section, with a simple theoretical analysis of the mechanism of separation.

\section{EXCLUSION MECHANISM}

In the following, we assume that any energetic interaction between the polymer and the substrate can be neglected; the is, the molecular adsorption or desorption with the stationary phase is insignificant Only size exclusion is considered. This is justified for large polymer molecules since surface contact interaction is small compared with the volume effect.

All molecules will flow through the intestitial volume regardless of size. For the internal volume, we will assume it consists of system of cylindrical channels with radius $d$. Partition of polymers between the internal volume and the external volume is due to a difference in entropy in these two regions. Inside the pores, due to their finite size, molecular motion is restricted and the entropy becomes lower. Let $k$ be the ratio of the concentrations inside and outside the pores,

$$
k=\frac{C_{\text {in }}}{C_{\text {owt }}} .
$$

Then

$$
-R T \ln k=\Delta G^{\circ}=-T \Delta S^{0}
$$

since no energy difference exists between these two regions.

From Eq. (1) the volumetric distribution ratio $K$ is

$$
K=\frac{V_{i}-V_{0}}{V_{i}} .
$$

Taking $V_{c}-V_{0}$ to be the effective volume the polymer can freely occupy inside the pore, $V_{\text {.ff }}$

$$
K=-\frac{V_{\text {fff }}}{V_{i}}=\frac{C_{\text {in }}}{C_{\text {iff }}}
$$

with

$$
C_{i n}=\frac{N}{\tilde{V}_{i}^{-}}, \quad C_{s f f}=\frac{N}{V_{e j f}}
$$

where $N$ is the number of polymers with molecular weight $M$ that are inside the pores. Since no energetic difference exists between the outside interstial volume and the effective volume inside the pores,

$$
C_{\text {sff }}=C_{\text {oxt }}
$$

und one has

$$
K=\frac{C_{\text {in }}}{C_{\text {out }}}=k .
$$

According to the Boltzman relation

$$
S^{\circ}=R \ln -\frac{W_{\text {in }}}{W_{\text {owt }}} .
$$

From Eq. (3) through Eq. (7), one has

$$
K=W_{\text {in }}
$$

where $W_{i n}$ is the probability distribution per unit volume inside a channel of radius $d, W_{\text {ost }}$ is the probability distribution for polymer in the same volume without channel restriction.

Consider the polymer to be a sphere of root-mean-square radius $\bar{r}$, then

$$
K=\frac{W_{\text {in }}}{W_{\text {oxt }}}=\frac{(d-\bar{r})^{z}}{d^{2}} .
$$

The actual value of $K$ should be a little larger than that given by Eq. (9) because the polymer can distort itself to fiit into the channel. The polymer mean radius is related to molecular weight ${ }^{\prime)}$ as

$$
\bar{r}=a M^{\jmath}
$$

with $a$ and $\delta$ determined by the polymer structure and solvent.

From Eqs. (9), (10) one expects

$$
1-\sqrt{K}=\frac{\vec{r}}{d}=\frac{a}{d} M^{3} .
$$

Thus, a plot of $\log (1-\sqrt{K})$ vs. $\log M$ should be close to a straight line. In next 
section, we will examine seven sets of experimental data to show that the linear relation holds over most of the range of the data.

\section{RESULTS}

There are many articles on GPC calibration in the literature, most of them do. not list all the raw data needed. We selected the data of Haller') and that of Van Kreveld and Van den Hoed') for a comparison of the following two calibration procedures:

(A) Haller's procedure, plot of $\log$ $(1-K)$ vs. $\log M$.

(B) Our procedure, plot of $\log (1-\sqrt{K})$ vs. $\log M$.

In Haller's paper, commercial dextran fractions were chromatographed on controlled pore glass. The eluant was a $0.1 M$ glycine buffer. Five sets of data for different pore diameters (84 $\AA$ to $517 \AA$ ) were used. The $\log (1-\sqrt{ } K)$ vs. $\log M$ plot is shown in Fig. 1 , and the $\log (1-K)$ vs. $\log M$ plot is shown in Fig. 2 for comparison. In the experiment of Van Krevold et al.", two columns were used: Porasil $C$ (mean pore diameter $330 \AA$ ) and Porasil D (mean pore diameter $700 \AA$ ). They used them to separate Polystyrene standards of narrow molecular weight distribution. The eluants

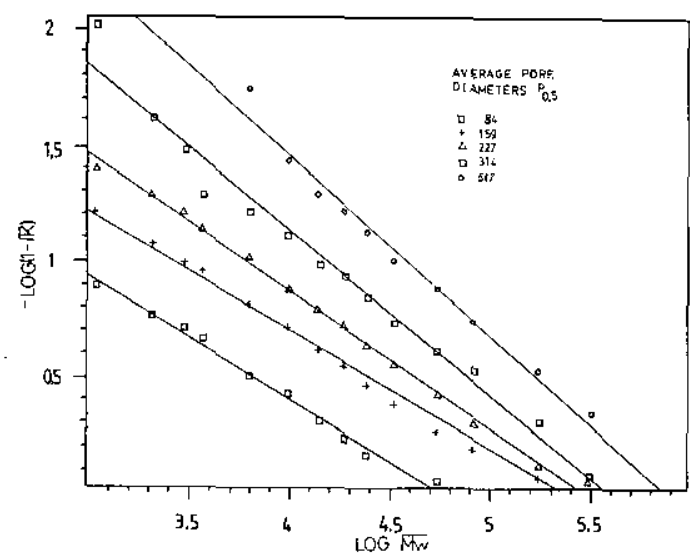

Fig. 1. $\log (1-\sqrt{K})$ versus $\log M$ plot for dextran fractionation by controlled pore glass. Data from reference (4).

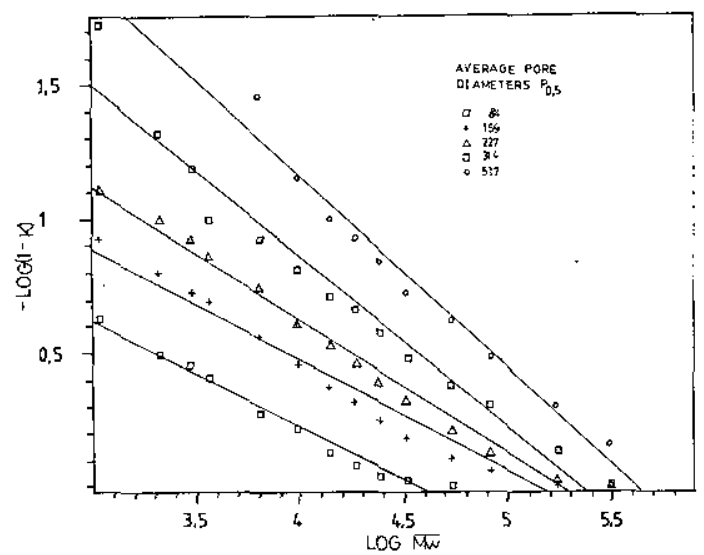

Fig. 2, $\log (1-K)$ versus $\log M$ plot for dextran fractionation by controlled pore glass. Data from reference (4).

is Tetrahydrofuran containing $0.025 \%$ Ionol as antioxidant. We chose these data because the mean radii of gyration are also available. The resulting calibration plots are shown in Fig. 3 and Fig. 4.

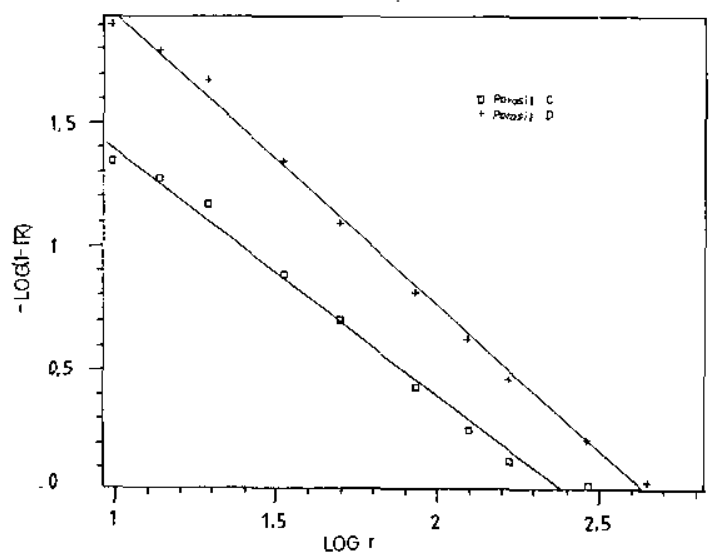

Fig. 3. $\log (1-\sqrt{K})$ versus $\log M$ plot for polystyrene fractionation by Porasil glass. Data from reference (8).

One can see that for the seven sets of data, our procedure gives a better linear plot when compared to the $\log (1-K)$ vs. $\log M$ plot. The standard deviations of the linear regression analyses are given in Table 1. 


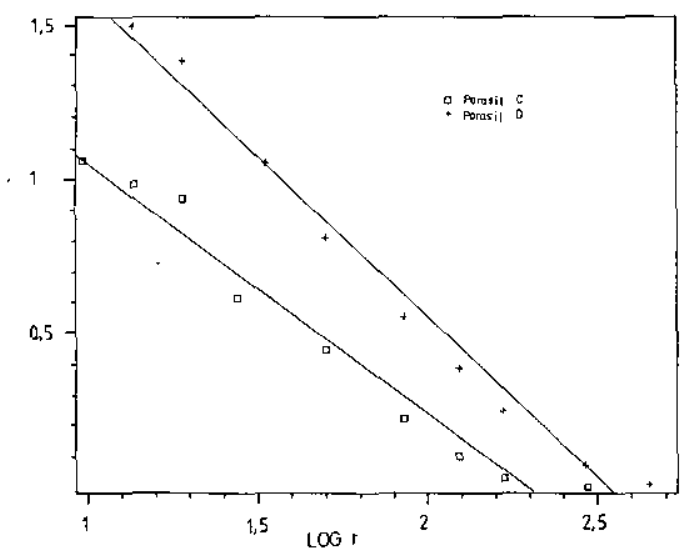

F g. 4. $\log (1-K)$ versus $\log M$ plot for polystyrene fractionation by Porasil glass. Data from reference (8).

\section{DISCUSSION}

Our calibration procedure is based on a separation theory in which a rigid spherical shape for polymer is assumed. This will fail when special affinity exists between the polymer and the column material. Therefore; strictly speaking, this procedure should apply only when size exclusion is the main mechanism. It is best when applied to controlled pore glass and hydrophobic polymers. One should be more careful when the glass surface is modified chemically or a strong hydrophillic effect is present. However, Spatarico and Beyer" demonstrated that the concept of hydrodynamic-volume still applies to four markedly different hydrophillic polymers

Table 1. Comparison of Calibration Curves

A. Dextran/controlled glass

\begin{tabular}{c|c|c|c|c|c|c|c|c}
$\begin{array}{c}\text { Pore diameter } \\
\text { (A) }\end{array}$ & $\begin{array}{c}\text { No. of data } \\
\text { points }\end{array}$ & \multicolumn{4}{|c|}{ Our method } & \multicolumn{3}{|c}{ Haller's method } \\
\hline 517 & 12 & 4.51 & 0.77 & $5.7 \times 10^{-2}$ & 3.98 & 0.71 & $6.4 \times 10^{-2}$ \\
314 & 14 & 3.94 & 0.71 & $7.5 \times 10^{-2}$ & 3.38 & 0.64 & $8.9 \times 10^{-2}$ \\
227 & 14 & 3.21 & 0.59 & $2.6 \times 10^{-2}$ & 2.58 & 0.49 & $4.7 \times 10^{-2}$ \\
159 & 14 & 2.75 & 0.52 & $4.3 \times 10^{-2}$ & 2.11 & 0.41 & $6.1 \times 10^{-2}$ \\
84 & 11 & 2.51 & 0.53 & $2.4 \times 10^{-2}$ & 1.80 & 0.40 & $3.2 \times 10^{-2}$ \\
\hline
\end{tabular}

B. Polystyrene/Porasil

\begin{tabular}{|c|c|c|c|c|c|c|c|}
\hline \multirow{2}{*}{$\begin{array}{c}\text { Pore diameter } \\
\text { (A) }\end{array}$} & \multirow{2}{*}{$\begin{array}{l}\text { No. of data } \\
\text { points }\end{array}$} & \multicolumn{3}{|c|}{ Our method } & \multicolumn{3}{|c|}{ Haller's method } \\
\hline & & $\begin{array}{l}\log \\
M \text { intercept }\end{array}$ & -slope & $\begin{array}{l}\text { standard } \\
\text { deviation }\end{array}$ & $M$ intercept & -slope & $\begin{array}{l}\text { standard } \\
\text { deviation }\end{array}$ \\
\hline 700 & 10 & 3.07 & 1.17 & $3.64 \times 10^{-2}$ & 2.61 & 1.04 & $6.64 \times 10^{-8}$ \\
\hline 330 & 9 & 2.34 & 0.98 & $4.83 \times 10^{-2}$ & 1.84 & 0.81 & $6.47 \times 10^{-2}$ \\
\hline
\end{tabular}

as long as no strong adsorption to the glass occurs. In the later case, the separation in any event would not be good.

The range of $K$ values we have used for the calibration curves is from $K=0.013$ to $K=0.98$, essentially the full range. One notes that at large $M$ the linearity is not as good. This could be due the existence of some larger pores and the flexibility of the polymer configuration. If we discount the last few points at large $M$, the linear fit will be even better. For a practical procedure, we suggest limiting the linear plot to $K$ values greater than $K=0.15$; at lower $K$ the deviation from linearity becomes progressively larger. Theoretically, one expects the slopes of the $\log (1-\sqrt{K})$ vs. $M$ (or $r$ ) plot should be constant for the same column but different pore diameters. For an ideal polymer near 
the $\theta$ point $\delta$ should be in the range $0.5-0.6$. From Fig. 1, the slopes indeed generally fall in this range. But they are definitely not constant. We suggest treating the slope as an empirical parameter.

Also, we note that the intercept point on the molecular weight axis should represent the critical permeation size. That is, it corresponds to the largest size of the polymer that can pass through the pore. The critical permeation size obtained from this procedure is somewhat larger thun that obtained from Haller's procedure.

In conclusion, the hypothesis that GPC separation is mainly controlled by size exclusion from a mean effective pore appears correct. A model based on this hypothesis gives a new calibration procedure with a straightforward linear plot. The procedure is practical in routine polymer fractionation by GPC.

Acknowledgement: We thank Miss Jin-Ru Fan for preparing the manuscript. The work is partiaily support by National Science Council of the Republic of China.

\section{NOMENCLATURES}

V. elution volume

V. intestitial volume
$V_{i} \quad$ internal volume

$K$ distribution ratio

$k \quad$ equilibrium constant

$W_{\text {in }}$ probability distribution inside channel

$W_{\text {out }}$ probability distribution without channel restriction

$d$ channel radius

$\bar{r}$ root-mean-square radius of the polymer

\section{REFERENCES}

(1) T. Kremmer and L. Boross, Gel Chromatography, John Wiley and Sons, New York, Chapter 4, (1979).

(2) P. Andrews, Molecular-Sieve Chromatography, Brit. Med. Bull., 22, 109 (1965).

(3) H. Determonn, Gel Chromatography, SpringerVerlag, Heidelberg, p. 63 (1969).

(4) W. Haller, Macromolecule, 10, 83 (1977).

(5) A. M. Basedow, K. M. Ebert, H. J. Ederer and E. Fosshog, J. Chromatogr., 192, 259 (1980).

(6) W. Haller, H. G. Gschwender and K. R. Peters, J. Chromatogr., 211, 53 (1981).

(7) H. Yamakawo, Modern Theory of Polymer Solutions, Harper and Row, New York, p. 72 (1971).

(8) M. E. Van Kreveld and N. Van den Hoed, $J$. Chromatography, 83, 111 (1973).

(9) A. L. Spatorico and G. L. Beyer, J. Appl. Poly. Sci., 19, 2933 (1975).

(Received August 14, 1984) 